\title{
FOLDER EDUCATIVO SOBRE DOAÇÃO DE LEITE MATERNO DURANTE A PANDEMIA DA COVID-19
}

\section{EDUCATIONAL FOLDER OF BREAST MILK DONATION DURING THE COVID-19 PANDEMIC}

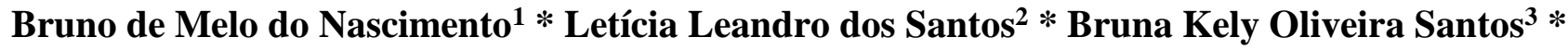 Antonia Lucileide Andrade da Cunha ${ }^{4}$ Natasha Marques Frota ${ }^{5}$ Anne Fayma Lopes Chaves $^{6}$}

RESUMO

O objetivo deste estudo foi descrever o processo de elaboração de um folder educativo sobre doação de leite durante a pandemia de Covid-19. Pesquisa metodológica que envolveu as etapas: levantamento bibliográfico e a elaboração do material educativo, os quais foram realizados no mês junho de 2020. Foi feito uma busca na Biblioteca Virtual em Saúde da Rede Global de Bancos de Leite Humano e no Portal de Boas Práticas em Saúde da Mulher, da Criança e do Adolescente da Fiocruz. Realizou-se uma revisão integrativa na qual foi abordada a situação das doações de leite humano no contexto da Covid-19 e as recomendações estabelecidas. O folder intitulado "Doar para Ajudar", foi construído no programa CANVA, sendo utilizado tons neutros com uma linguagem simples e ilustrações representativas. O folder ajudará na conscientização da doação de leite materno durante a pandemia de Covid-19, sendo este o primeiro material com esse enfoque específico.

Palavras-chave: Educação em Saúde; Bancos de Leite; Aleitamento Materno; Infecções por Coronavírus; Tecnologia Educacional.

\begin{abstract}
The aim of this study was to build an educational folder to raise awareness of milk donation during the Covid-19 pandemic. Methodological research that involved the steps: bibliographic survey and the preparation of educational material, which were carried out in June 2020. A search was made in the Virtual Health Library of the Global Network of Human Milk Banks and in the Good Practices Portal in Fiocruz's Women, Children and Adolescents' Health. An integrative review was carried out in which the situation of human milk donations was addressed in the context of Covid-19 and the established recommendations. The folder entitled "Donate to Help" was built in the CANVA program, using neutral tones with simple language and representative illustrations.
\end{abstract}

Keywords: Health Education; Milk Banks; Breastfeeding; Coronavirus Ifections; Educational Technology.

\footnotetext{
${ }^{1}$ Graduando em Enfermagem pela Universidade da Integração Internacional da Lusofonia Afro-Brasileira (UNILAB). Membro do Grupo de Pesquisa e Extensão em Saúde Sexual e Reprodutiva da UNILAB.

${ }^{2}$ Graduanda em Enfermagem pela Universidade da Integração Internacional da Lusofonia Afro-Brasileira (UNILAB). Membro do Grupo de Pesquisa e Extensão em Saúde Sexual e Reprodutiva da UNILAB.

${ }^{3}$ Graduanda em Enfermagem pela Universidade da Integração Internacional da Lusofonia Afro-Brasileira (UNILAB). Membro do Grupo de Pesquisa e Extensão em Saúde Sexual e Reprodutiva da UNILAB.

${ }^{4}$ Graduanda em Enfermagem pela Universidade da Integração Internacional da Lusofonia Afro-Brasileira (UNILAB). Membro do Grupo de Pesquisa e Extensão em Saúde Sexual e Reprodutiva da UNILAB

${ }^{5}$ Doutora em Enfermagem pela Universidade Federal do Ceará. Docente do Curso de Enfermagem da Universidade da Integração Internacional da Lusofonia Afro-Brasileira (UNILAB). Coordenadora do Grupo de Estudo e Pesquisa em Saúde do Idoso da UNILAB.

${ }^{6}$ Doutora em Enfermagem pela Universidade Federal do Ceará. Docente do Curso de Enfermagem da Universidade da Integração Internacional da Lusofonia Afro-Brasileira (UNILAB). Co-líder do Núcleo de Estudos e Pesquisas em Promoção da Saúde Sexual e Reprodutiva da Universidade Federal do Ceará. Coordenadora do Grupo de Pesquisa e Extensão em Saúde Sexual e Reprodutiva da UNILAB. Email: annefayma@yahoo.com.br
} 


\section{INTRODUÇÃO}

O mundo enfrenta a doença respiratória causada pelo vírus coronavírus SARS-CoV, a Covid-19, que se disseminou de forma rápida, gerando uma pandemia, a qual provocou um abalo nos serviços de saúde de países pouco estruturados, com mínima capacidade de vigilância e de pesquisa ${ }^{(1)}$. No contexto da saúde materno-infantil não há evidências científicas que comprovem a transmissão vertical do SARS-CoV, tendo em vista que estudo realizado em Wuhan na China evidenciou não haver presença do vírus nas amostras de líquido amniótico, sangue do cordão umbilical, leite materno e swab da orofaringe do recém-nascido ${ }^{(2)}$. Logo, a Organização Pan-Americana da Saúde recomenda que a amamentação seja mantida em caso de infecção pela Covid-19 $9^{(3)}$.

Devido a rápida disseminação do vírus algumas medidas foram adotadas nas unidades de saúde visando garantir à saúde do recém-nascido (RN) no contexto da pandemia: unidades neonatais e Banco de Leite Humano (BLH) não devem ser fechados nem reduzidos, no entanto, cuidados devem ser adotados para a prevenção de aglomerações, realização de triagem para sintomatologia respiratória e síndrome gripal para pais e mães que visitam bebês em unidades neonatais e apoio para a extração de leite materno $^{(4)}$.
No entanto, devido a recomendação do distanciamento social, juntamente com o medo de contrair o vírus, evidências apontam uma redução significativa de doações de leite. Entre janeiro e abril de 2019, foram 61.146 doações de leite materno ${ }^{(5)}$, em 2020, no mesmo período, o número caiu para 58.155. Esses dados alertam os BLH para o risco da falta do leite materno (LM), cabendo a este serviço atuar no intuito de incentivar, proteger e promover o aleitamento materno (AM), visando a diminuição dos índices de morbimortalidade infantil ${ }^{(6)}$.

As evidências científicas apontam que em crianças menores de um ano acometidas com Covid-19 as taxas de complicações graves são mais altas do que em crianças mais velhas, sendo necessário que profissionais de saúde continuem incentivando a doação de leite para que o LM seja disponibilizado as crianças que necessitam, promovendo seus benefícios e ajudando na recuperação da saúde ${ }^{(7)}$.

Nesse contexto, torna-se imprescindível o uso de tecnologias educacionais, as quais são ferramentas úteis e facilitadoras na construção do processo de ensinoaprendizagem ${ }^{(8)}$. Desse modo, vislumbrou-se a criação de um folder, haja vista que evidências apontam que essa modalidade de tecnologia proporciona uma linguagem acessível, otimiza o conhecimento, proporciona maior agilidade e compreensão 
do processo de cuidar e cuidado ${ }^{(9)}$.

A construção do folder poderá contribuir para uma melhora dos índices de doação de leite materno, já que facilitará a disseminação de informações científicas sobre o assunto, visando a conscientização e o incentivo das mulheres a realizar a doação, o que pode influenciar positivamente na saúde materno-infantil. Assim, o objetivo deste estudo foi descrever o processo de elaboração de um folder educativo sobre doação de leite durante a pandemia de Covid-19.
Para a construção do material educativo adotou-se as seguintes etapas ${ }^{(10)}$ : submissão do projeto ao comitê de ética em pesquisa; levantamento bibliográfico; elaboração do material educativo. Para o presente estudo, utilizou-se apenas o levantamento bibliográfico e a elaboração do material educativo, os quais foram realizados no período de maio e junho de 2020 (Figura $1)$.

\section{MÉTODO}

Figura 1 - Fluxograma sobre a construção do material educativo. Fortaleza, Ceará, Brasil, 2020.

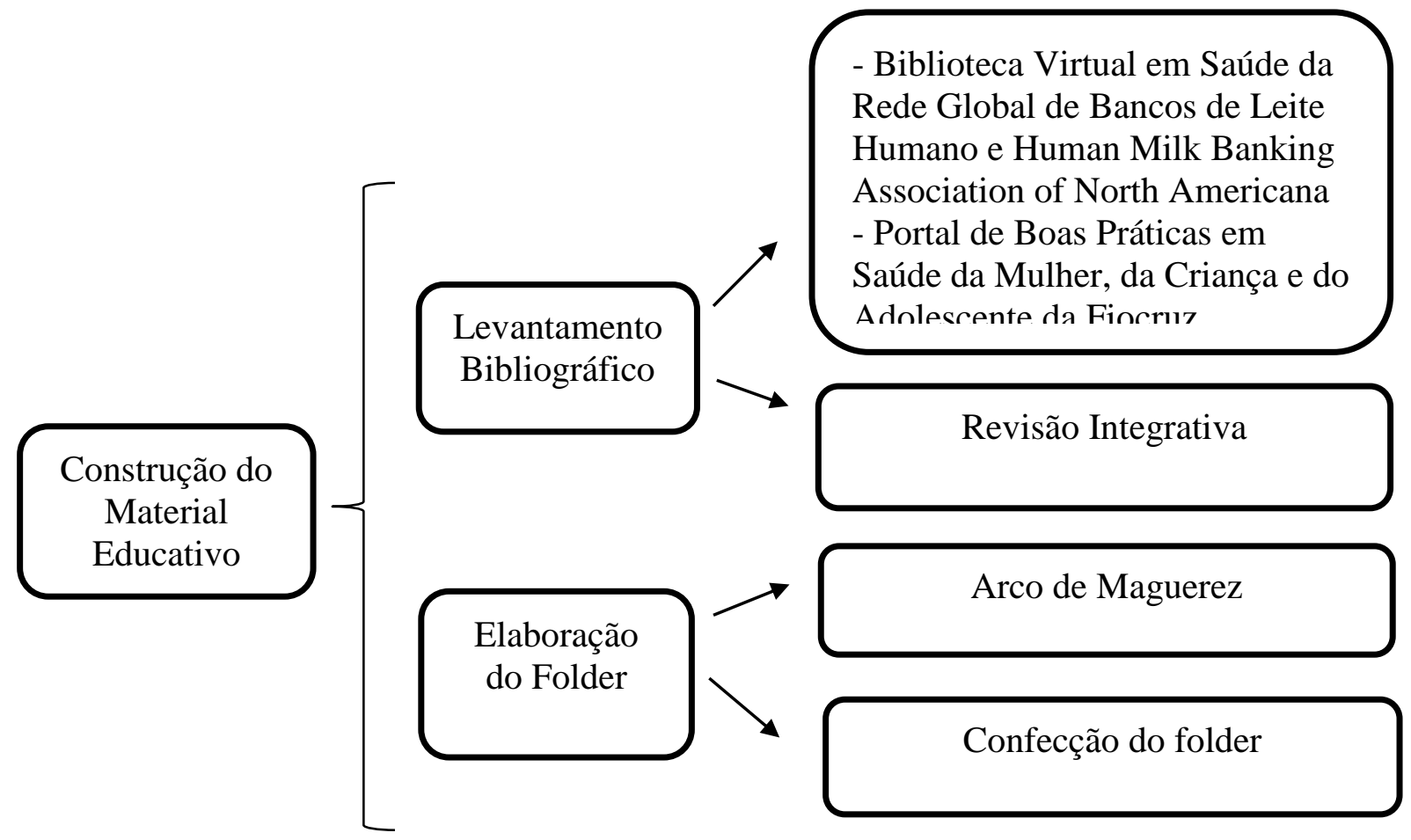

Fonte: elaborado pelos autores. 
Diante do contexto da pandemia, o MS e os BLH mundiais traçaram recomendações específicas para essas instituições, as quais devem ser seguidas pelos profissionais, pacientes e familiares. Desse modo, na etapa do levantamento bibliográfico optou em consultar a Biblioteca Virtual em Saúde da Rede Global de Bancos de Leite Humano, a Biblioteca Virtual da Human Milk Banking Association of North American e o Portal de Boas Práticas em Saúde da Mulher, da Criança e do Adolescente da Fiocruz, órgão vinculado ao Ministério da Saúde.

Em junho de 2020 foi realizada uma revisão integrativa nas bases de dados: Literatura Latino-americana e do Caribe em Ciências da Saúde (LILACS), Scientific Electronic Library Online (SCIELO), e PUBMED, tendo como pergunta norteadora: Qual a situação das doações de leite humano no contexto da Covid-19 e que recomendações estão sendo implementadas para dar continuidade a esse processo?

Os critérios de inclusão para estabelecer a amostra foram: documentos do tipo material informativo ou artigos científicos, disponíveis eletronicamente na íntegra, divulgados nos idiomas português, inglês e espanhol. Foram excluídos os artigos que não responderam à pergunta norteadora.

Utilizou-se os descritores controlados dos Descritores em Ciência da Saúde (DeCS) "Leite humano", "Banco de leite",
"Coronavírus" e "Pandemia" e as do Medical Subject Headings (MeSH) foram "Milk human", "Milk Bank", "Coronavírus" e "Pandemic", sendo realizado o cruzamento de todos os descritores citados em cada base de dados utilizando o operador booleano and.

Para extração dos dados dos estudos foi utilizado o instrumento adaptado de Ursi ${ }^{(11)}$, sendo excluídas as variavéis não analisadas nessa pesquisa e incluído o desfecho sobre as recomendações. As publicações foram avaliadas segundo a classificação proposta por Melnyk e Fineout-Overholt ${ }^{(12)}$. A etapa de seleção, extração e análise dos resultados dos estudos foram realizadas por dois revisores que trabalharam de forma independente.

$\mathrm{Na}$ segunda etapa foi realizada a elaboração do folder a qual teve como referência o arco de Maguerez, que consiste em uma metodologia problematizadora que fornece um caminho para a atuação sobre os problemas da realidade. A mesma é dividida em cinco partes, sendo elas: observação da realidade, pontos-chave, teorização, hipóteses de soluções e aplicação à realidade ${ }^{(13)}$. $\mathrm{Na}$ hipótese de soluções, vislumbra-se que a elaboração do folder educativo irá auxiliar na compreensão da necessidade e continuidade da doação de leite humano. A aplicação na realidade será realizada em outro momento, tendo em vista que essa pesquisa se propôs apenas à construção do folder educativo.

A confecção do folder seguiu os passos 
descritos por Rodrigues e Carvalho ${ }^{(14)}$, os quais afirmam que $o$ folder deve ter quantidade mínima de dobras, contendo nele algumas imagens atrativas e dá ênfase às ideias mais interessantes com quadros ou palavras em fontes maiores.

O folder foi construído na medida da folha 1005 X $591 \mathrm{~mm}$. O texto foi escrito com fontes disponíveis no programa CANVA. Em relação ao tamanho das letras, foram utilizadas as seguintes medidas: título 6,0, tópicos com perguntas 5 e respostas 4 . O espaçamento entre linhas foi de 1,0. Foram escolhidos tons neutros para facilitar a leitura e não tornar cansativa.

Um folder com linguagem simples e de fácil entendimento se faz imprescindível no cenário atual. Foram selecionados os possíveis questionamentos das nutrizes para elucidar os seus receios frente a segurança e necessidade da doação. Ademais, o folder é formado por perguntas e respostas, uma ilustração lateral e uma frase com teor de incentivo.

\section{RESULTADOS}

A construção do folder educativo intitulado "Doar para Ajudar", foi ilustrada para fornecer orientações e incentivo a doação de leite materno no contexto atual da pandemia de Covid-19 e seguiu dois passos: levantamento bibliográfico e elaboração do folder.

\section{Levantamento Bibliográfico}

$\mathrm{O}$ primeiro passo do processo de construção do folder consistiu no levantamento bibliográfico (Quadro 1). Na Biblioteca Virtual em Saúde da Rede Global de Bancos de Leite Humano foi encontrado o documento-Aleitamento Materno em tempos de Covid-19-recomendações na maternidade e após a alta, a qual aborda as recomendações para mulheres com sintomas de síndrome gripal, infecção respiratória ou confirmação de caso de SARS- CoV-2, que queiram doar leite ${ }^{(15)}$.

. Na Biblioteca Virtual da Human Milk Banking Association of North American foram selecionados os seguintes documentos: Banking and COVID-19, que são recomendações sobre como deve ser feito a seleção de doadores de leite; e o Statement on Pasteurized Donor Human Milk and Covid-19 by the USBC Donor Milk Constellation, que tem por objetivo explanar sobre promover a educação em saúde direcionada a atuação ao Covid-19 frente a doação de leite materno ${ }^{(16-}$ 17)

No Portal de Boas Práticas em Saúde da Mulher, da Criança e do Adolescente da Fiocruz, na qual foi selecionada a nota técnica $\mathrm{N}^{\mathrm{o}}$ $8 / 2020$ COCAM/CGCIVI/DAPES/SAPS/MS que tem como objetivo descrever as recomendações para doação de leite materno 
QUADRO 1 - Dados relevantes para a construção do folder. Fortaleza, Ceará, Brasil, 2020.

\begin{tabular}{|c|c|}
\hline TÍTULO & ORIENTAÇÕES \\
\hline $\begin{array}{l}\text { Aleitamento } \\
\text { Materno } \\
\text { em tempos de } \\
\text { COVID-19 - } \\
\text { recomendações na } \\
\text { maternidade e após } \\
\text { a alta. }\end{array}$ & $\begin{array}{l}\text { Recomenda-se a contraindicação da doação de leite maternoaos } \\
\text { bancos de leite em casos que mães que estejam com sintomas gripais, } \\
\text { infecção respiratória ou confirmação de caso de SARS-CoV-2, além das que } \\
\text { tiveram contatos domiciliares de casos com síndrome gripal ou caso } \\
\text { confirmado de SARS-CoV-2. } \\
\text { Ressalta-se que após ser curada da infecção a doação de leite poderá ser } \\
\text { retomada. }\end{array}$ \\
\hline $\begin{array}{l}\text { Milk Banking and } \\
\text { COVID-19 }\end{array}$ & $\begin{array}{l}\text { Recomendações sobre o processo de seleção de possíveis doadoras } \\
\text { de leite materno: solicitação do exame de sangue e a aplicação de um } \\
\text { questionário para verificar detalhadamente viagens internacionais da doadora } \\
\text { e seu histórico recente de doença e de } \\
\text { seus familiares. }\end{array}$ \\
\hline $\begin{array}{l}\text { Statement on } \\
\text { Pasteurized Donor } \\
\text { Human Milk and } \\
\text { COVID-19 by } \\
\text { the USBC Donor } \\
\text { Milk Constellation }\end{array}$ & $\begin{array}{l}\text { Retrata a importância da educação em saúde no contexto do } \\
\text { Covid-19, sendo um meio que favorece a propagação de informações } \\
\text { baseadas em evidência sobre a doação de leite materno. }\end{array}$ \\
\hline
\end{tabular}

Fonte: elaborado pelos autores.

Esse levantamento foi fundamental, visto que os profissionais de saúde seguem essas recomendações técnicas científicas para desempenhar suas funções. Observou-se a realização de rastreamento da mulher doadora, considerando os sintomas gripais, contatos domiciliares e histórico de viagens. Ficou evidente a contraindicação da doação de leite materno em casos de mães com Covid-19, no entanto, sendo enfatizado a possível doação após cura.

$\mathrm{Na}$ análise da revisão integrativa, durante a seleção dos estudos, procedeu-se a busca na base de dados no qual foram encontrados 18 artigos, sendo doze na base de dados PUBMED, cinco na LILACS e um na 
SciELO. Destes, foram excluídos: um por não estar disponível eletronicamente e gratuitamente, onze por não responderem à pergunta norteadora. Portanto, a amostra final desta revisão integrativa foi constituída por seis artigos, sendo todos com nível de evidência 7.

Foram elencadas as principais informações extraídas dos documentos incluídos na revisão integrativa (Quadro 2).

Quadro 2 - Apresentação da síntese dos artigos incluídos na revisão integrativa. Fortaleza, Ceará, Brasil, 2020.

\begin{tabular}{|c|c|c|}
\hline Título & Recomendação & Método \\
\hline $\begin{array}{l}\text { Fonoaudiologia, } \\
\text { amamentaçãoe } \\
\text { COVID-19: } \\
\text { Informações aos } \\
\text { fonoaudiólogos }{ }^{(19)}{ }^{\text {a }}\end{array}$ & $\begin{array}{l}\text { Armazenamento de } \\
\text { leite para doação }\end{array}$ & $\begin{array}{l}\text { Doadoras devem realizar desinfecção } \\
\text { dos frascos com soluções sanitárias } \\
\text { adequadas, antes de manusear, mesmo } \\
\text { que não apresentem sintomatologia de } \\
\text { infecção respiratória. }\end{array}$ \\
\hline $\begin{array}{l}\text { Use of Disinfectant } \\
\text { Wipes to Sanitize } \\
\text { Milk's Containers of } \\
\text { Human Milk Bank } \\
\text { During COVID-19 } \\
\text { Pandemic }\end{array}$ & $\begin{array}{lr}\text { Uso de } & \text { lenços } \\
\text { desinfetantes } & \text { em } \\
\text { recipientes } & \text { que } \\
\text { armazenam leite doado }\end{array}$ & $\begin{array}{l}\text { Uso de lenços desinfetantes, } \\
\text { contendo } 20 \mathrm{ml} \text { de hipoclorito de } \\
\text { sódio a } 0,1 \% \text {. Estima-se que com um } \\
\text { único pano úmido, é possível } \\
\text { desinfetar a parte externa e a } \\
\text { superfície de } 55 \text { recipientes em cerca } \\
\text { de } 15 \text { minutos. }\end{array}$ \\
\hline $\begin{array}{l}\text { International } \\
\text { Perspectives } \\
\text { Concerning Donor } \\
\text { Milk Banking During } \\
\text { the SARS-CoV-2 } \\
\text { (COVID-19) } \\
\text { Pandemic }^{(21)}\end{array}$ & $\begin{array}{lr}\text { Segurança } & \text { no } \\
\text { processo de coleta, } \\
\text { armazenamento } \\
\text { fornecimento do leite } \\
\text { materno doado. }\end{array}$ & $\begin{array}{l}\text { Avaliar a temperatura da doadora, } \\
\text { preenchimento de um questionário, } \\
\text { incluindo perguntas sobre o contanto } \\
\text { nos últimos } 14 \text { dias com pessoas de } \\
\text { locais com alta incidência. Coleta } \\
\text { realizada no domicílio. Rejeição de } \\
\text { doação de pessoas que tiveram sido } \\
\text { expostas ao vírus nas últimas duas } \\
\text { semanas e de pessoas que foram } \\
\text { testados positivo para Covid-19, } \\
\text { podendo retornar após tenha sido feito }\end{array}$ \\
\hline
\end{tabular}




\begin{tabular}{|c|c|c|}
\hline & & $\begin{array}{l}\text { uma cultura com resultado } \\
\text { negativo. }\end{array}$ \\
\hline $\begin{array}{l}\text { Maintaining a Viable } \\
\text { Donor Milk Supply } \\
\text { During the SARS- } \\
\text { CoV-2 (COVID-19) } \\
\text { Pandemic }\end{array}$ & $\begin{array}{l}\text { Medidas utilizadas } \\
\text { na triagem de doadores } \\
\text { de leite. }\end{array}$ & $\begin{array}{l}\text { As doadoras são obrigadas a fornecer } \\
\text { um relatório sobre viagens ao exterior e } \\
\text { doenças recentes, incluindo todos os } \\
\text { membros da família em casa. As } \\
\text { doações são adiadas por } 28 \text { dias em } \\
\text { caso de história de sintomas } \\
\text { respiratórios ou qualquer contato com } \\
\text { casos/suspeitos de Covid-19 são } \\
\text { adiadas temporariamente. }\end{array}$ \\
\hline $\begin{array}{l}\text { US NICUs and donor } \\
\text { milk banks brace for } \\
\text { COVID-19 }\end{array}$ & $\begin{array}{l}\text { Estocagem } \\
\text { racionamento } \\
\text { preventivo do leite } \\
\text { materno }\end{array}$ & $\begin{array}{l}\text { O leite doado em excedente é } \\
\text { armazenado e alocado somente a bebês } \\
\text { prematuros em situação de alto risco. }\end{array}$ \\
\hline $\begin{array}{l}\text { Maintaining safety } \\
\text { and service provision } \\
\text { in human milk } \\
\text { banking: a call to } \\
\text { action in response to } \\
\text { the COVID- } \\
19 \text { pandemic }\end{array}$ & $\begin{array}{l}\text { Direcionamento } \\
\text { adequado do leite } \\
\text { materno }\end{array}$ & $\begin{array}{l}\text { Reduzir a quantidade de tempo de } \\
\text { separação da mãe com COVID-19 e } \\
\text { seu bebê e apoiar o uso do leite } \\
\text { materno da própria mãe, } \\
\text { consequentemente o excesso de } \\
\text { demanda por leite doador diminuirá. }\end{array}$ \\
\hline
\end{tabular}

Fonte: elaborado pelos autores.

A partir dessa revisão foi visto que duas evidências apontam a importância dos cuidados necessários em relação a desinfecção dos frascos para as doadoras de 
leite, três apontaram a necessidade de educação em saúde para o rastreamento das doadoras em relação a Covid-19, por meio de questionários, aferição de temperatura e exames, bem como a contraindicação da doação em casos positivos e a orientação para adiar a doação.

\section{Elaboração do Folder}

Para construção do folder, foi seguido o arco de Maguerez, no qual a observação da realidade foi impossibilitada devido ao contexto da pandemia Covid-19, logo, a análise foi feita através de dados do MS a respeito da diminuição das doações de leite para as unidades ${ }^{(13)}$. No ponto-chave foram traçados aspectos que precisam ser trabalhados para solucionar o problema, a saber: produção de informações para o esclarecimento de lactantes potenciais doadoras, visando empoderar na doação e, consequentemente, elevar os números de doações.

A teorização consistiu na necessidade de alterar os comportamentos das lactantes, para que ocorra adequação ao contexto social atual, contribuindo para a melhoria do conhecimento a respeito das recomendações frente a Covid-19 e o processo de amamentação, através de linguagem facilitada por ilustrações e imagens.

A figura 2 apresenta a síntese das ideias do folder: 1. Título e o conteúdo enfatizando o contexto da amamentação e da doação de leite materno na pandemia da Covid-19; 2. Conteúdo sobre os cuidados necessários durante a extração e doação de leite, bem como a importância da doação para a saúde materno-infantil; 3. Frase motivacional para incentivo a doação; 4. Ilustração criada pelos autores fazendo alusão a amamentação e doação de leite.

Figura 2 - Apresentação da síntese das ideias contidas no folder. Fortaleza, Ceará, Brasil, 2020.

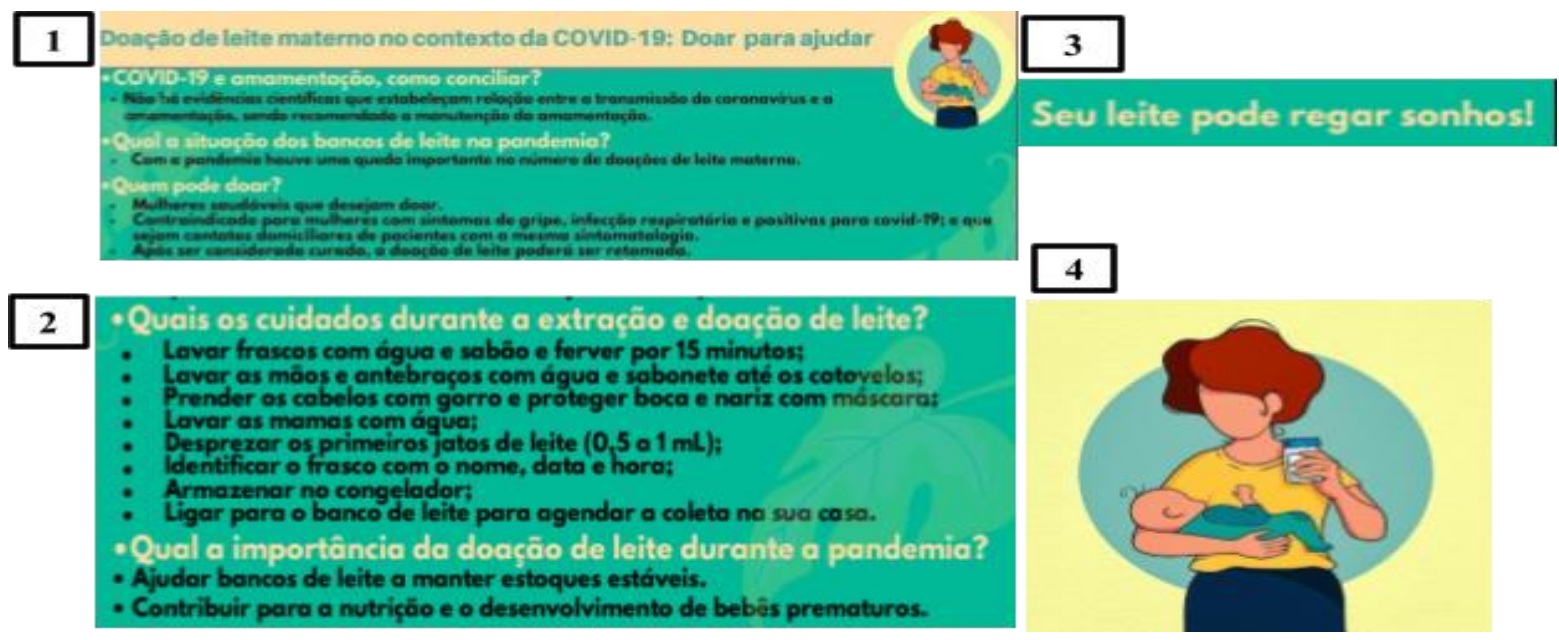

Fonte: elaborado pelos autores. 
O material tem sua versão final livremente nas redes sociais e por vias conforme figura 5, visando ser difundido impressas.

Figura 3 - Folder educativo para conscientização da doação de leite materno durante a pandemia de Covid-19. Fortaleza, Ceará, Brasil, 2020.

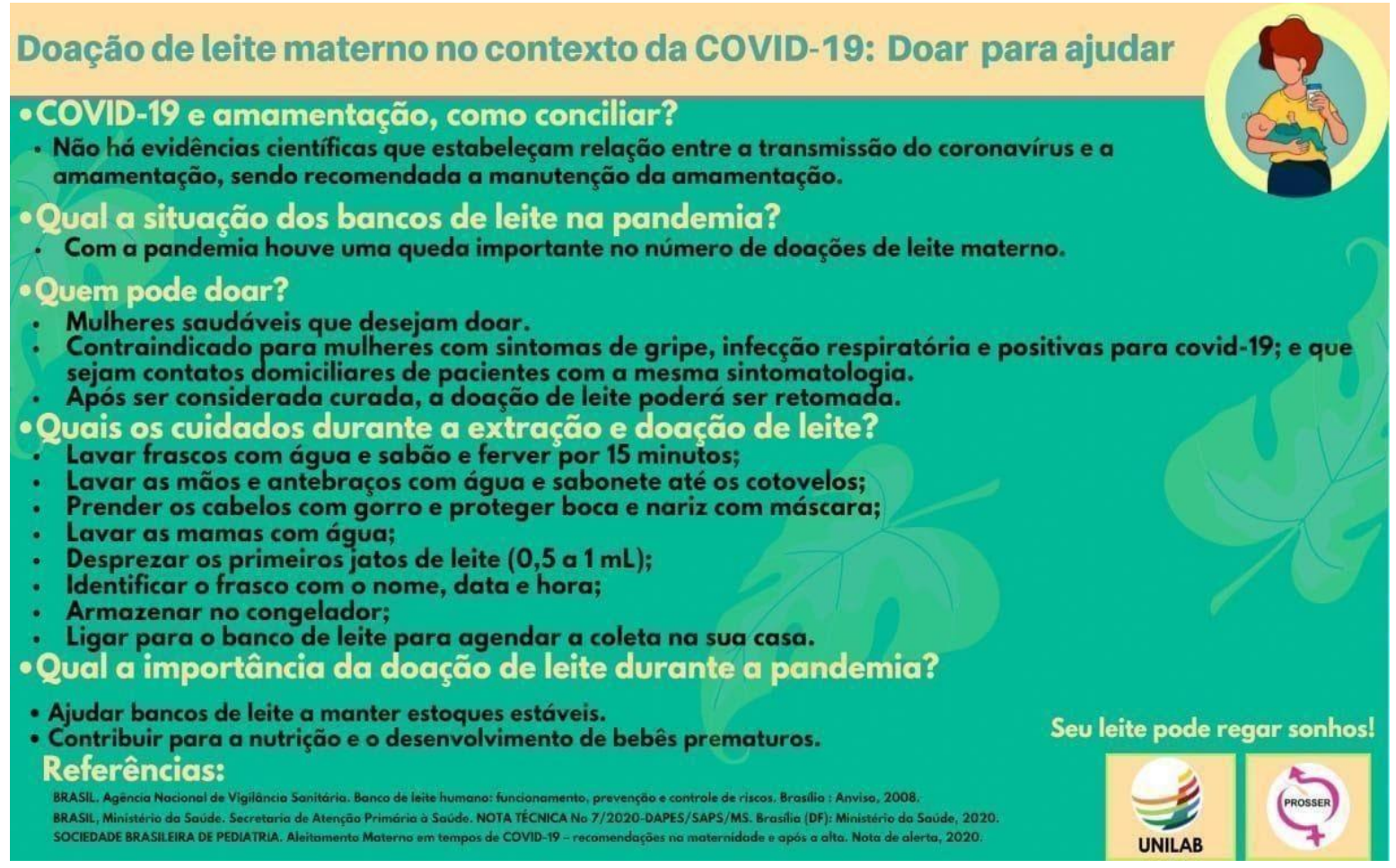

Fonte: elaborado pelos autores.

\section{DISCUSSÃO}

A pandemia da Covid-19 vem acompanhada de mudanças e impactos no comportamento social, exigindo estratégias específicas de planejamento das ações do BLH a curto, médio e longo prazo para manutenção da amamentação, e principalmente, da captação de leite para manter $\mathrm{O}$ atendimento na Unidade de Tratamento Intensivo Neonatal (UTIN) ${ }^{(25)}$.

Corroborando com os achados do levanto bibliográfico, pesquisa que envolveu gestores de BLH apontou a importância da educação em saúde as candidatas a doação de leite materno, como forma de reforçar as orientações no contexto da pandemia, principalmente em relação a coleta domiciliar diante do isolamento social ${ }^{(25)}$.

Ficou evidente nos estudos analisados a contraindicação da doação de leite materno em casos confirmados de mães com Covid19 , concordando com a RDC $\mathrm{n}^{\mathrm{o}} 171 / 2006^{\text {(26) }}$ 
a qual descreve a necessidade de a mulher apresentar-se saudável para essa ação. Apesar de não existirem evidências consolidadas da transmissão vertical do SARSCoV-2 no leite materno, a Sociedade Brasileira de Pediatria também reforça essa contraindicação, enfatizando que a doação poderá ser retomada após cura ${ }^{(15)}$.

$\mathrm{Na}$ revisão integrativa foi evidenciado os cuidados necessários em relação à desinfecção dos frascos para as doadoras de leite, visando a prevenção da disseminação do vírus. A Rede Brasileira de Bancos de Leite Humano em suas recomendações para acolhimento e manejo clínico em AM as lactantes assintomáticas ou sintomáticas de Covid-19 instituiu medidas de biossegurança: utilização de equipamentos de proteção individual pela equipe, evitar aglomerações, suspender os grupos educativos; após o atendimento solicitar que a equipe realize a desinfecção com álcool $70 \%$ da cadeira; acessórios da bomba elétrica ou manual devem ser direcionados para lavagem e esterilização imediatamente após o uso; e ao fim do processo de extração de leite realizar a desinfecção da superfície externa dos frascos com álcool $70 \%$ antes de armazená-los no freezer ${ }^{(27)}$.

Em um estudo realizado na cidade do Rio de Janeiro ${ }^{(28)}$, ressaltou a importância de as mães manterem a prática da amamentação durante o período de seis meses exclusivos, com vistas a proporcionar tanto os benefícios para seu filho como diminuir a demanda de leite do BLH. Desse modo, o BLH além de realizar o controle de qualidade do leite, ainda permite a construção de uma rede de possibilidade relacionadas a nucleação familiar, de forma a favorecer a aquisição do leite humano pelas mães impossibilitadas de amamentar, contribuindo para a melhor interação entre elas e suas crianças.

No contexto da pandemia, os BLH recorreram a novas estratégias para driblar o distanciamento social, sendo utilizado os meios eletrônicos, grupos de aplicativos, atendimento virtual, folhetos e folders mostrando-se métodos eficazes nas ações a curto prazo ${ }^{(25)}$.

Em diversas áreas percebe-se uma reorganização com a utilização de diferentes estratégias na prestação de cuidados durante a pandemia. Estudo reflexivo de natureza qualitativa apontou que no âmbito da Atenção Primária à Saúde os folders podem ser um grande aliado na prestação de cuidados pois permitem a disseminação de informações importantes ${ }^{(29)}$.

No contexto do BLH ainda é visto lacunas na criação de tecnologias educativas que abordem a pandemia da Covid-19. No entanto, deve ser incentivado a criação desse tipo de tecnologia de fácil acesso e que transmita informações essenciais, haja vista que essa utilização pode favorecer a 
divulgação e adesão ao serviço especializado do BLH, o qual contribui de forma satisfatória e efetiva para a promoção do AM, impactando em melhores taxas de duração do AM e exclusivo ${ }^{(30)}$.

Pressupõe-se que o folder facilitará a elucidação das hesitações das possíveis doadoras. Pela linguagem simples e breve, por ser visualmente atrativo e pela praticidade de seu compartilhamento, isso auxiliará no melhor entendimento das informações de saúde que visam contribuir para o aumento das doações de leite materno.

A contribuição desta pesquisa está pautada nas necessidades de apoio a doação de leite frente a pandemia da Covid-19, no sentido de incentivar esta experiência mediante a nova realidade que a população está inserida. Pois acredita-se que o uso de tecnologias educativas facilite a adesão das mulheres aos seus respectivos planos de cuidados, bem como no incentivo à doação de leite para os BLH.

\section{CONCLUSÃO}

O objetivo do estudo de construir um folder educativo para conscientização da doação de leite materno durante a pandemia de Covid-19 foi alcançado, sendo este o primeiro material com esse enfoque específico. Foi elaborada uma tecnologia educativa de fácil acesso e distribuição, visando orientar, conscientizar e motivar as nutrizes para a prática de doação de leite humano aos BLH no período de pandemia da Covid-19.

Reforça-se que foi importante criar, desenvolver e produzir um material de qualidade com linguagem simples e visualmente atrativo, o qual irá auxiliar no melhor entendimento das informações de saúde que visam contribuir para o aumento das doações de leite.

Como limitação para a construção dessa tecnologia, observou-se a carência de pesquisas com grande nível de evidência científica para compor a revisão, como ensaios clínicos randomizados e revisões sistemáticas. Pretende-se futuramente realizar a validação desta tecnologia com juízes, tornando-a confiável e válida.

\section{REFERÊNCIAS}

1. Worldometer. Pandemia de coronavírus [Internet]. [atualizado em: 11 dez. 2020; acesso em: 12 mai. 2020]. Disponível em: https://www.worldometers.info/

2. Chen H, Guo J, Wang C, Luo F, Yu $\mathrm{X}$, Zhang W, et al. Clinical characteristics and intrauterine vertical transmission potential of COVID-19 infection in nine pregnant women: a retrospective review of medical records. The Lancet. Março de 2020;395(10226):809-15. Disponível em: https://www.thelancet.com/journals/lancet/arti cle/PIIS0140-6736(20)30360-3/fulltext

3. Ministério da Saúde (BR). Secretaria de atenção primária à saúde. Nota Técnica $\quad \mathrm{n}^{\circ}$ 10/2020- 
COCAM/CGCIVI/DAPES/SAPS/MS

[Internet]:Brasília, Ministério da Saúde; 2020 [acesso em 19 jun 2020].

Disponível

em:

https://rblh.fiocruz.br/sites/rblh.fiocruz.br/file s/usuario/80/notatecnica102020coc\%20amcgc ividapessapsms_003.pdf

4. Secretaria de Estado de Saúde, Departamento de Ações em Saúde. Orientações relacionadas à Saúde da Criança durante a Pandemia de COVID-19 [Internet]. Rio Grande do Sul (BR): Secretaria de Saúde. [atualizado em: 27 de out. de 2020; acesso em: 01 de dez. 2020]. Disponível em: https://coronavirus.rs.gov.br/upload/arquivos/ 202010/27175400-orientacoes-relacionadas-asaude-da-crianca-durante-a-pandemia-decovid-19.pdf

5. $\quad$ Fundação Oswaldo Cruz. Hospitais pedem doações de leite materno; estoque está abaixo do ideal. [Internet]. [acesso em: 12 jun. 2020].

Disponível em:

https://rblh.fiocruz.br/hospitais-pedem-

doacoes-de-leite-materno-estoque-esta-

\%20abaixo-do-ideal

6. Maternidade-Escola Assis Chateaubriand. Banco de Leite Humano [Internet]. Fortaleza (BR): MEAC; [acesso em: 15 de jun. 2020]. Disponível em: http://www2.ebserh.gov.br/web/meac$\underline{\text { ufc/banco-de-leite-humano-blh }}$

7. Eastin C, Eastin T. Epidemiological characteristics of 2143 pediatric patients with 2019 coronavirus disease in China. The Journal of Emergency Medicine. Abril de 2020;58(4):712-3. Disponível em: https://www.jem-journal.com/article/S07364679(20)30271-7/fulltext

8. Maniva SJC de F, Carvalho ZM de F, Gomes RKG, Carvalho REFL de, Ximenes LB, Freitas CHA de. Educational technologies for health education on stroke: an integrative review. Rev Bras Enferm [Internet]. 2018 [acesso em: 14 de jun. de 2020];71(suppl
4):1724-31. Disponível em: https://www.scielo.br/scielo.php?script=sci_a bstract\&pid=S0034-

71672018001001724\&lng=pt\&nrm=iso\&tlng $=$ en

9. Severino CDO. Universidade Federal de Santa Catarina. Construção de um folder ilustrado: tecnologia de educação para uso correto de medicamentos por usuários de uma unidade de pronto atendimento. 2014;24.

10. Echer IC. Elaboração de manuais de orientação para o cuidado em saúde. Rev Latino-Am Enfermagem [Internet]. Outubro de 2005 [acesso em: 20 de jun. de 2020];13(5):754-7. Disponível em: https://www.scielo.br/scielo.php?script=sci_ar ttext\&pid=S0104-

$11692005000500022 \& \operatorname{lng}=$ en \&nrm=iso\&tlng $=\mathrm{pt}$

11. Ursi ES, Gavão CM. Prevenção de lesões de pele no perioperatório: revisão integrativa da literatura. Rev Latino-Am Enfermagem [Internet]. Fevereiro de 2006 [acesso em: 12 de jun. de 2020];14(1):124-31. Disponível

em:

https://www.scielo.br/scielo.php?script=sci_ar ttext\&pid=S0104-

$11692006000100017 \& \operatorname{lng}=$ en \&nrm=iso\&tlng $=\mathrm{pt}$

12. Fineout-Overholt E. MB. Evidencebased practice in nursing \& healthcare: A guide to best practice. Philadelphia: Lippincott Williams \& Wilkins; 2011.

13. Sistema Universidade Aberta do SUS. UNASUS| Processo Educativo em Saúde [Internet] Santa Catarina (BR); [atualizado em: 9 Dez 2020; acesso em 21. jun 2020]. Disponível em:

https://unasus2.moodle.ufsc.br/course/view.p hp?id=49

14. Rodrigues MAN, Carvalho ADP. O gênero textual folder a serviço da educação 
ambiental. REGET. 31 de agosto de 2014;18(2):982-9.

15. Brasileira de Pediatria. Departamento Científico de Aleitamento Materno [Internet]. 22 de maio 2020 [22 de jun. 2020]. Disponível em: https://www.sbp.com.br/imprensa/detalhe/nid/ aleitamento-materno-em-tempos-de-covid-19recomendacoes-na-maternidade-e-apos-a-alta/

16. Human Milk Banking Association of North America. MILK Banking and COVID19. Full statement on milk banking and COVID-19. [Internet] 3 jun. 2020 [acesso em 15 jun. 2020]. Disponível em: https://www.hmbana.org/

17. United States Breastfeeding Committee USBC. Statement on Pasteurized Donor Human Milk \& COVID-19 [Internet]. 5 mai. 2020 [acesso em: 12 de junho de 2020]. Disponível em: http://www.usbreastfeeding.org/p/cm/ld/fid=8 $95 \&$ tid $=889 \& \operatorname{sid}=1624$

18. Ministério da Saúde (BR). Secretaria de atenção primária à saúde. Nota Técnica ${ }^{\circ}$ 8/2020-COCAM/CGCIVI/DAPES/SAPS/MS [Internet]:Brasília, Ministério da Saúde; 2020 [acesso em 19 jun 2020]. Disponível em: https://www.unasus.gov.br/especial/covid19/p $\underline{\mathrm{df} / 96}$

19. Miranda VSG de, Rech RS, Maahs MAP, Berbert MCB, Almeida ST de. Fonoaudiologia, amamentação e COVID-19: informações aos fonoaudiólogos. CoDAS [Internet]. 2020 [acesso em: 12 de junho de 2020];32(3):e20200124.

20. De Rose DU, Reposi MP, Amadio P, Auriti C, Dall'Oglio I, Corsetti T, et al. Use of Disinfectant Wipes to Sanitize Milk's Containers of Human Milk Bank During COVID-19 Pandemic. J Hum Lact [Internet]. Agosto de 2020 [acesso em: 13 de jun. De

2020];36(3):547-9. Disponível em: http://journals.sagepub.com/doi/10.1177/0890 $\underline{334420924639}$

21. Marinelli KA. International Perspectives Concerning Donor Milk Banking During the SARS-CoV-2 (COVID-19) Pandemic. J Hum Lact [Internet]. Agosto de 2020 [acesso em: 12 de jun. de 2020];36(3):492-7. Disponível

em: http://journals.sagepub.com/doi/10.1177/0890 334420917661

22. Jayagobi PA, Mei Chien C. Maintaining a Viable Donor Milk Supply During the SARS-CoV-2 (COVID-19) Pandemic. J Hum Lact [Internet]. Novembro de 2020 [acesso em: 12 de jun. de 2020];36(4):622-3. Disponível

em: http://journals.sagepub.com/doi/10.1177/0890 $\underline{334420931828}$

23. Furlow B. US NICUs and donor milk banks brace for COVID-19. The Lancet Child \& Adolescent Health [Internet]. Maio de 2020 [acesso em: 12 de junho de 2020];4(5):355. Disponível em:

https://linkinghub.elsevier.com/retrieve/pii/S2 $\underline{352464220301036}$

24. Shenker N, Aprigio J, Arslanoglu S, Aye NSS, Bærug A, Bar Yam N, et al. Maintaining safety and service provision in human milk banking: a call to action in response to the COVID-19 pandemic. The Lancet Child \& Adolescent Health [Internet]. Julho de 2020 [acesso em: 12 de jul. de 2020];4(7):484-5. Disponível em: https://linkinghub.elsevier.com/retrieve/pii/S2 352464220301346

25. Marchiori GRS, Alves VH, Pereira AV, Vieira BDG, Rodrigues DP, Dulfe PAM, et al. Ações da enfermagem nos bancos de leite humano em tempos de COVID-19. Rev Bras Enferm [Internet]. 2020 [acesso em: 14 de jun. de 2020];73(suppl 2):e20200381. Disponível

em: https://www.scielo.br/scielo.php?script=sci_ar ttext\&pid=S0034- 
$\underline{71672020001400155 \& \operatorname{lng}=\text { en\&nrm=iso\&tlng }}$ $=\mathrm{pt}$

26. Ministério da Saúde (BR). Resolução RDC/ANVISA $\mathrm{n}^{\circ} 171$, de 4 de setembro de 2006. Dispõe sobre o regulamento Técnico para o funcionamento de Bancos de Leite Humano [Internet]. 2006[acesso em: 13 de abr. 2020]. Disponível em: http://www.redeblh.fiocruz.br/media/rdc_171. pdf

27. Fundación Oswaldo Cruz. Recomendación No.03/20.160420: Recomendaciones para Acogimiento y Manejo Clínico en lactancia materna de gestantes, puérperas y lactantes asintomáticas o sintomáticas de COVID-19 por el Banco de Leche Humana. [acesso em 13 jun. 2020]. Disponível em: https://rblh.fiocruz.br/sites/rblh.fiocruz.br/file s/usuario/77/covid-19__rblh_reco mendacao_n.0320.160420_espanhol.pdf

28. Alves J de S, Oliveira MIC de, Rito RVVF. Orientações sobre amamentação na atenção básica de saúde e associação com o aleitamento materno exclusivo. Ciênc Saúde Coletiva [Internet]. Abril de 2018 [acesso em: 12 de dezembro de 2020];23(4):1077-88. Disponível em:

https://www.scielo.br/scielo.php?script=sci_ar ttext\&pid=S1413-

$\underline{81232018000401077 \& \operatorname{lng}=e n \& n r m=i s o \& t \operatorname{lng}}$ $=\mathrm{pt}$

29. Associação Brasileira de Enfermagem, Departamento de Enfermagem na Atenção Básica. Enfermagem na atenção básica no contexto da COVID-19 [Internet]. Brasília, DF: ABen/DEAB; 2020. [acesso em; 13 de jun. 2020]. 87p. Disponível em: http://www.abennacional.org.br/site/wpcontent/uploads/2020/09/E-BOOKATENCAO-BASICA.pdf

30. Oliveira AM, Lima LC, Costa CC, Chagas ACMA, Oriá MOB, Chaves AFL. Duração do aleitamento materno em mulheres atendidas no banco de leite humano. Rev Enferm Atual In Derme [Internet]. 26 de agosto de 2020 [acesso em: 14 de ago. 2020];93(31). Disponível em: https://revistaenfermagematual.com.br/index. php/revista/article/view/663

Submissão: 2021-03-11

Aprovado: 2021-03-31 